\title{
EFFECT OF SURGICALLY ASSISTED RAPID MAXILLARY EXPANSION ON THE NASAL AIRWAYS
}

\author{
Arafa Gad Allah Ibrahim*, Mansour Mohammad Hussein**, Mohammad Abd Elhamied Shuman***
}

\begin{abstract}
Objectives: Transverse maxillary deficiency is a common problem among patients seeking orthodontics care. It is characterized by a narrow maxilla in relation to the rest of the craniofacial structures, a narrow palatal vault, and often a posterior cross bite. Aim: The aim of the present study was to evaluate the effect of surgically assisted rapid maxillary expansion on the nasal airways to measure outcome of nasofibroscopy in evaluation of nasal airways and to measure the changes in area of the nasal cavities. Patients and Methods: The present study was carried out on nine patients' healthy adult. Surgical assisted rapid maxillary expansion (SARME) was done for each patient. All patients were assessed by nasofibroscopy for increasing in nasal cavities through the expansion period. Results: Results of the current study have indicated that nasofibroscopy values of surgical assisted rapid maxillary expansion were higher along the observation periods. The nasofibroscopy results showed increasing in nasal cavities. Conclusion: these results support the view that surgical assisted rapid maxillary expansion is effective in increasing of nasal cavities through the follow up period.
\end{abstract}

Keywords: surgically assisted rapid maxillary expansion, nasopharyngeal, nasofibroscopy

\section{INTRODUCTION}

Transverse maxillary deficiency is a common problem among patients seeking orthodontics care. It is characterized by a narrow maxilla in relation to the rest of the craniofacial structures, a narrow palatal vault, and often a posterior cross bite $^{(1,2)}$. Maxillary transverse deficiency (MTD) is a pathological condition that may be associated with other types of dentoskeletal alterations, with esthetic and functional implications, including respiratory problems. The incidence is around $3-18 \%$ in orthodontic patients. Patients with this condition have a narrow nasal cavity, which increases the resistance to nasal airway flow ${ }^{(3-6)}$.

Surgically assisted rapid maxillary expansion (SARME) as recommended by Bell and Epker. is a well-established method for correction of transverse maxillary deficits in nongrowing patients. Crossbite and crowded teeth are a typical characteristic of maxillary compression syndromes. Nasofibroscopy has revolutionized the diagnostic method nowadays for buccopharyngeal and nasopharyngeal regions. The nasofibroscopy, in addition to the ability to evaluate the nasopharyngeal region in three dimensions, is able to show a large variety of etiologic factors that cause the obstruction of the upper airway ${ }^{(7)}$.

\section{PATIENTS AND METHODS}

Patient Selection: Nine Patients were included in the present study. They were consecutive patients selected from the oral and maxillofacial surgery department that referred from orthodontic department Faculty of Dental Medicine, Al-Azhar University, Assuit branch. They were adult patients

\footnotetext{
* Assistant Lecturer of Oral and Maxillofacial Surgery, Faculty of Dental Medicine (Assiut) - Al-Azhar University

** Professor of Oral and Maxillofacial Surgery, Faculty of Dental Medicine (Cairo) Al-Azhar University

*** Assistant professor of Oral and Maxillofacial Surgery, Faculty of Dental Medicine - (Asiut Branch) Al-Azhar University
} 
of both sexes. All patients had maxillary transverse deficiency associated primarily with functional impairments, such as posterior uni- or bilateral cross bite, dental crowding, reduced nasal respiratory function or anterior-posterior skeletal anomalies and they were indicated for surgically assisted rapid maxillary expansion.

Preoperative evaluation, preparation and measurements: History was taken then all patients underwent complete physical examination. Clinical evaluation and examination by inspection and palpation of soft tissue

Nasofibroscopy exam; Nasofibroscopy measuring was performed by nasofibroscopy equipment (Fig.1). Nasofibroscopy performed in ENT department of Al-Azhar University Hospital, Assuit branch

A Hyrax device installation: Hyrax expansion appliance was applied before surgery and was inactivated. A rigid expansion appliance is usually cemented to the first premolars and first molars on each side.

Surgical procedure: Patients were prepared in general routine manner for operation under general anesthesia. Nasoendotracheal intubation was used in all cases, scrupulous disinfection of oral cavity and extraoral. After submucosal injection of vasoconstrictor intra oral incision will be made in the depth of the maxillary vestibule, $3-5 \mathrm{~mm}$ above the level of attached gingiva in the alveolar mucosa and leaving unattached mucosa on the alveolus to facilitate closure extended from second premolar on one side to second premolar in other side.

The soft tissues are reflected subperiosteally by periosteal elevator from the lateral aspect of the maxilla, with dissection to expose the anterior floor of the nose and piriform aperture area, posteriorly toward the pterygo-maxillary fissure. Osteotomy by using of surgical bur and the level of the lateral maxillary osteotomies is measured to be at least 5 $\mathrm{mm}$ above the apices of the teeth. As the anterior portion of the osteotomies is being performed, periosteal elevator is maintained in the piriform rim, lifting the nasal mucoperiosteum to protect it. The lateral wall osteotomies are extended posteriorly toward the pterygo-maxillary fissure (Fig. 2). On completion of the lateral maxillary osteotomy the osteotome is malleted posteriorly for approximately $30 \mathrm{~mm}$, to section the lateral nasal wall. Similar osteotomies are performed on the opposite side. The nasal spine and septum are now released with osteotome extending at least $30 \mathrm{~mm}$ posteriorly, having first performed an osteotomy below the nasal spine, leaving it attached to the septum and ensuring that the septum remains independent of the maxillary segments.

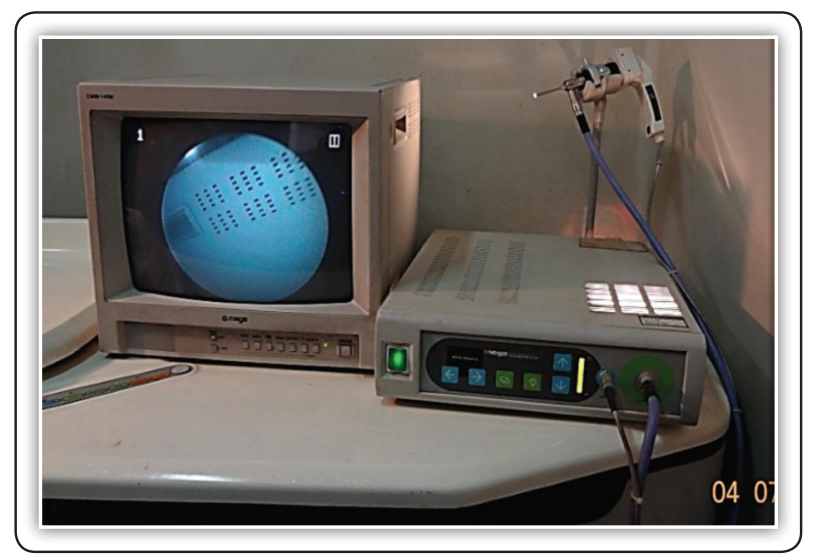

FIG (1) Photograph of equipment used to perform the nasofibroscopy.

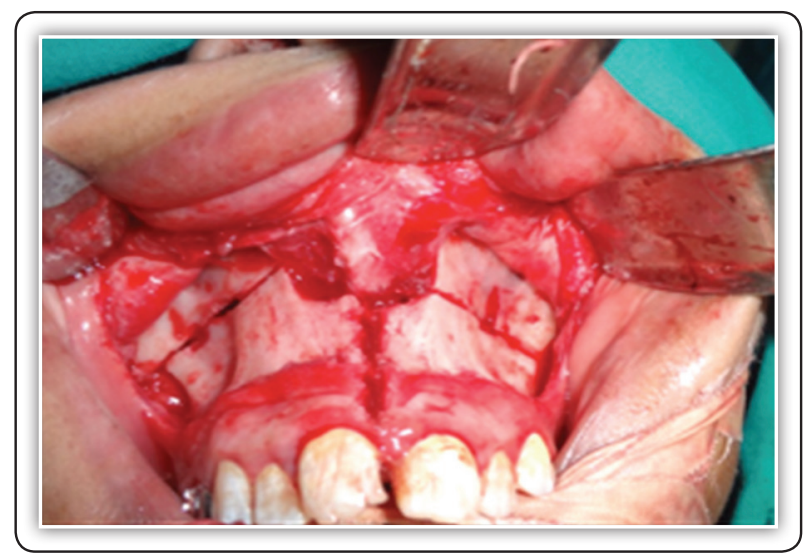

FIG (2) Photograph of bilateral osteotomy. 
Amidpalatal osteotomy is then accomplished by malletting an osteotome posteriorly, parallel to the palatal plane, into the intermaxillary suture and directed to the posterior nasal spine. These procedures ensure that separation of the entire midpalatal suture has occurred. Before the soft tissue incisions are closed, the expansion screw is turned eight to twelve times, so that the maxilla is expanded 2 to $3 \mathrm{~mm}$ this amount of immediate expansion.

Expansion protocol: Following the SARME procedure and after seven days' latency period and oral analgesic before expansion for controlling of pain the appliance is activated by the parents of patient, one millimetre each day, until the desired expansion has been achieved. It was expanded to its maximum width, because it is easier to allow the maxillary posterior segments to settle back, if necessary, into ideal transverse relationships with their lower opponents.

Postoperative Evaluation: Clinical evaluations were done at intervals of 2, 4, 7 and 15 days and directed toward the observation of the healing process, infection or any complications of wound healing.

Nasofibroscopy; Nasofibroscopy; was performed postoperatively to evaluate the effect of surgically assisted rapid maxillary expansion on the nasal airways immediately after expansion (T0), and 3-6 months after expansion T3, T4, T5, and T6 Detection of the nasal cavity's contours applying a geometric active contours function to identify object boundaries. Therefore, the nasal cavity was drawn by hand on the images yellow curve representing the initial contour before expansion and the red curves representing the active contours function after palatal expansion. Determination of nasal cavity antero-posterior diameter $(\mathrm{APg})$. At first a contouring of nasal cavity and two points were detected on the most right and left nasal cavity contour. from intersecting of those border lines, central line was derived, at last, the distance (APg) was defined by the cross points of the central lines with nasal cavity contour. Determination of nasal cavity width (dg). This distance was determined by perpendicular line to APg at it is mid-level. Finally, conversion of the measured data from pixels to millimeters by typography converter program

\section{RESULTS}

In all patients, the distractor device was well tolerated, remained stable, maintained the osteotomized segments during distraction in the desired alignment. Distraction was carried out manually and continued for 10 days without any difficulty.

Statistical analysis of nasofibroscopy findings: Using a software program SPSS statistical packaged for social sciences V19.0 for windows (SPSS INC, Chicago, USA). Data were presented as mean and standard deviation values. At the intervals of 3, 4,5, 6 , months with p-value of $(0.000)$ showed very high statistically significant table (1). The mean values of nasal cavity changes of right side for ANC and PNC from preoperative values at different intervals of the follow up period show highly statistically significant differences. figure (5).

TABLE (1): Mean Width changes(dg) for ANC.

\begin{tabular}{|c|c|c|c|c|}
\hline & Mean & Std.Dv & $\mathrm{t}$-Value & $\mathrm{P}$ \\
\hline \multirow{2}{*}{$\mathrm{T} 0$ - T3 } & 3.414 & 0.555 & \multirow{2}{*}{-13.779} & \multirow{2}{*}{0.000} \\
\hline & 4.117 & 0.469 & & \\
\hline \multirow{2}{*}{$\mathrm{T} 0$ - T4 } & 3.414 & 0.555 & \multirow{2}{*}{-16.282} & \multirow{2}{*}{0.000} \\
\hline & 4.066 & 0.491 & & \\
\hline \multirow{2}{*}{$\mathrm{T} 0$ - T5 } & 3.414 & 0.555 & \multirow{2}{*}{-9.400} & \multirow{2}{*}{0.000} \\
\hline & 3.936 & 0.380 & & \\
\hline \multirow{2}{*}{ T0 - T6 } & 3.414 & 0.555 & \multirow{2}{*}{-9.400} & \multirow{2}{*}{0.000} \\
\hline & 3.936 & 0.380 & & \\
\hline
\end{tabular}

* Significant at $P \leq 0.05$, 


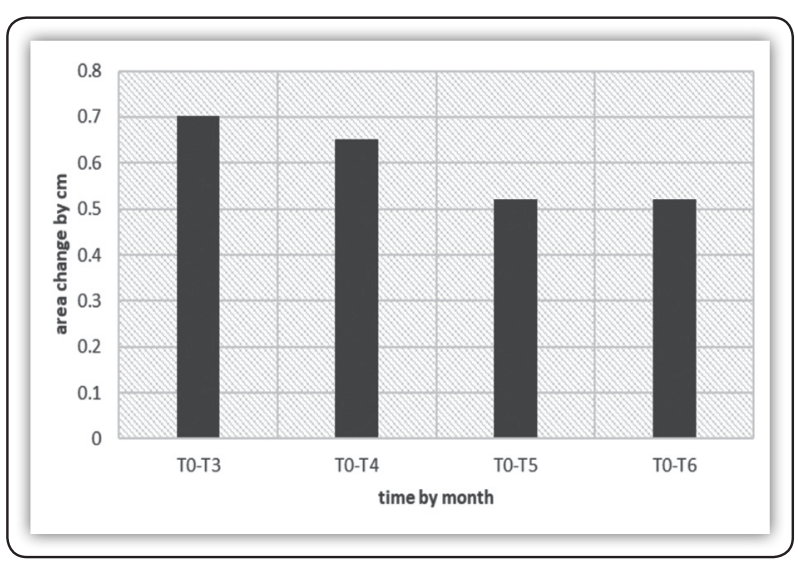

FIG (3) Bar-chart of nasal cavity change (ANC) from preoperative values at different intervals of the follow up period.

TABLE (2) Mean Anteroposterior diameter changes (Apg) for ANC.

\begin{tabular}{|c|c|c|c|c|}
\hline & Mean & Std.Dv & t-Value & $\mathrm{P}$ \\
\hline \multirow{2}{*}{$\mathrm{T} 0-\mathrm{T} 3$} & 3.929 & 0.390 & \multirow{2}{*}{-20.622} & \multirow{2}{*}{0.000} \\
\hline & 4.429 & 0.479 & & \\
\hline \multirow{2}{*}{$\mathrm{T} 0-\mathrm{T} 4$} & 3.929 & 0.390 & \multirow{2}{*}{-20.000} & \multirow{2}{*}{0.000} \\
\hline & 4.586 & 0.426 & & \\
\hline \multirow{2}{*}{$\mathrm{T} 0-\mathrm{T} 5$} & 3.929 & 0.390 & \multirow{2}{*}{-8.402} & \multirow{2}{*}{0.000} \\
\hline & 4.457 & 0.555 & & \\
\hline \multirow{2}{*}{ T0 - T6 } & 3.929 & 0.390 & \multirow{2}{*}{-9.285} & \multirow{2}{*}{0.000} \\
\hline & 4.443 & 0.489 & & \\
\hline
\end{tabular}

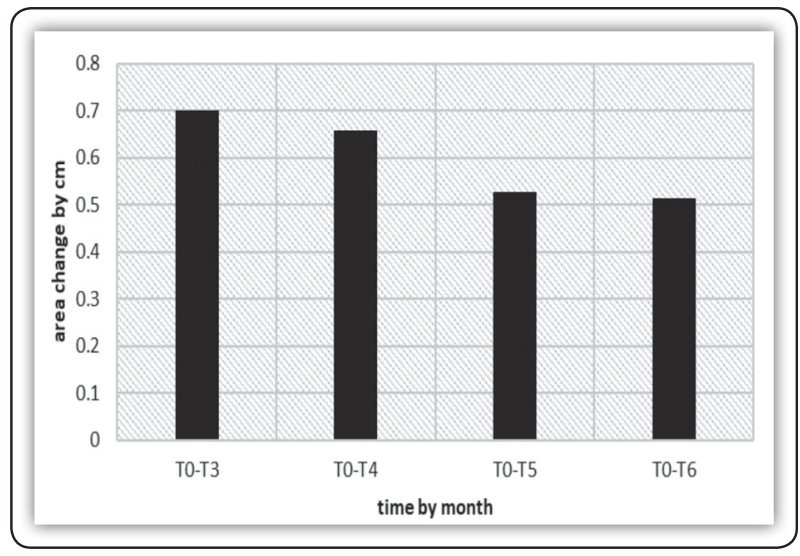

FIG (4) Bar-chart of nasal cavity change (ANC) from preoperative values at different intervals of the follow up period.
TABLE (3) Mean Width changes(dg) of the right side for PNC

\begin{tabular}{|c|c|c|c|c|}
\hline & Mean & Std.Dv & t-Value & P \\
\hline \multirow{2}{*}{ T0 - T3 } & 3.729 & 0.373 & \multirow{2}{*}{-13.856} & 0.000 \\
\cline { 2 - 3 } & 4.529 & 0.364 & & \\
\hline \multirow{2}{*}{ T0 - T4 } & 3.729 & 0.373 & \multirow{2}{*}{-4.515} & \multirow{2}{*}{0.000} \\
\cline { 2 - 3 } & 4.557 & 0.282 & & \\
\hline \multirow{2}{*}{ T0 - T5 } & 3.729 & 0.373 & \multirow{2}{*}{-13.664} & 0.000 \\
\cline { 2 - 3 } & 4.386 & 0.302 & & \multirow{2}{*}{0.000} \\
\hline \multirow{2}{*}{ T0 - T6 } & 3.729 & 0.373 & & \\
\cline { 2 - 3 } & 4.386 & 0.302 & & \\
\hline
\end{tabular}

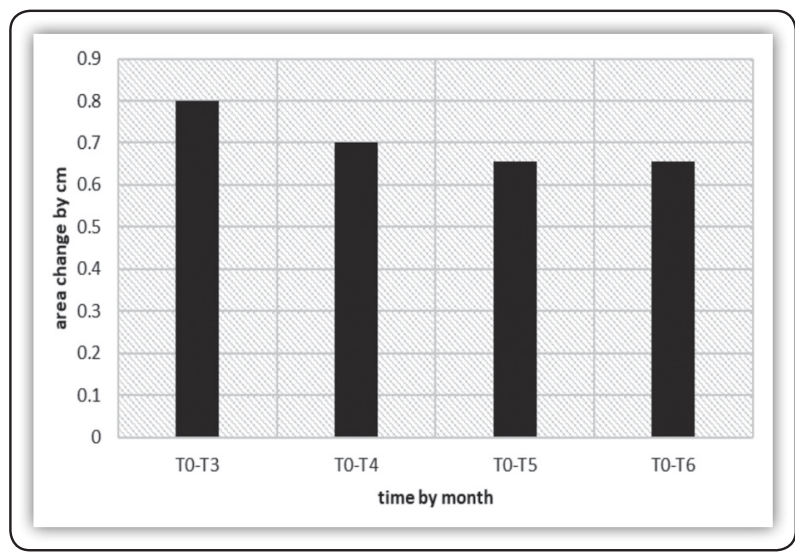

FIG (5) Bar-chart of nasal cavity change (PNC) from preoperative values at different intervals of the follow up period

TABLE (4) Mean Anteroposterior diameter changes (Apg) for PNC.

\begin{tabular}{|c|c|c|c|c|}
\hline & Mean & Std.Dv & t-Value & $\mathrm{P}$ \\
\hline \multirow{2}{*}{$\mathrm{T} 0-\mathrm{T} 3$} & 4.471 & 0.482 & \multirow{2}{*}{-20.721} & \multirow{2}{*}{0.000} \\
\hline & 5.343 & 0.513 & & \\
\hline \multirow{2}{*}{$\mathrm{T} 0-\mathrm{T} 4$} & 4.471 & 0.482 & \multirow{2}{*}{-10.331} & \multirow{2}{*}{0.000} \\
\hline & 5.214 & 0.474 & & \\
\hline \multirow{2}{*}{$\mathrm{T} 0-\mathrm{T} 5$} & 4.471 & 0.482 & \multirow{2}{*}{-13.664} & \multirow{2}{*}{0.000} \\
\hline & 5.129 & 0.461 & & \\
\hline \multirow{2}{*}{ T0 - T6 } & 4.471 & 0.482 & \multirow{2}{*}{-13.377} & \multirow{2}{*}{0.000} \\
\hline & 5.086 & 0.471 & & \\
\hline
\end{tabular}




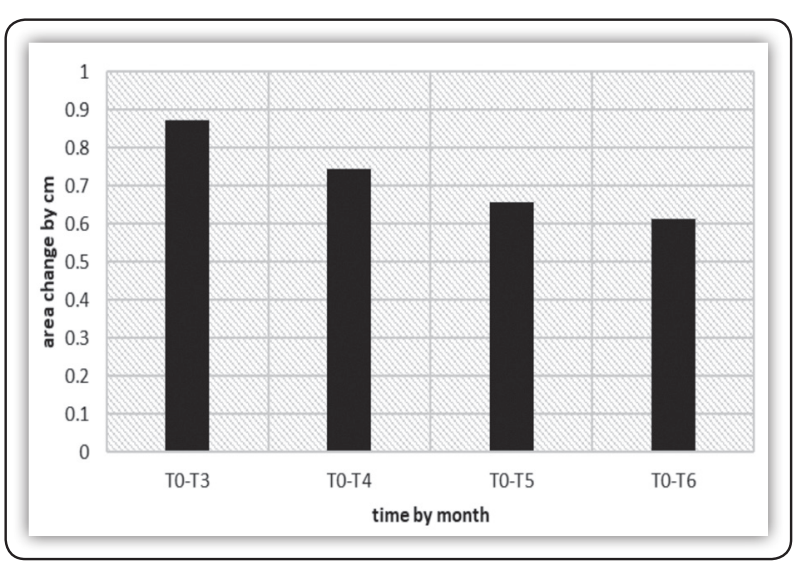

FIG (6) Bar-chart of nasal cavity change (PNC) from preoperative values at different intervals of the follow up period.

\section{DISCUSSION}

Various treatment protocols were proposed, all focusing on different aspects of the procedure. The lack of consensus from scientific studies puts clinicians in the challenging position of having to choose and balance between factors such as the complexity of the surgery, risks for the patient, expected long-term stability, the effect on soft tissue, effect on respiration, clinical accessibility for the procedure, etc. The treatment of the patients with this deficient has aimed to transversally increase the diameter of maxilla through orthodontic, orthopedic, and/or surgical procedures. In subjects with skeletal maturity, surgically assisted rapid maxillary expansion has showed good results. Surgically assisted rapid maxillary expansion became an overall accepted and common strategy for treatment of maxillary transverse deficiency in non-growing individuals in the late 1980's and early 1990's ${ }^{(8)}$. Surgically assisted rapid maxillary expansion with attachment of expansion appliances to the buccal teeth and osteotomy of the lateral maxillae and/ or the midpalatal suture has produced satisfactory results in adolescents and adults. Positive secondary effects include widening of the paranasal sinuses, preservation of the palatal arch configuration, and the opportunity to start multibracket therapy for alignment of the maxillary dental arch immediately on completion of the distraction period ${ }^{(9,10)}$. In the current study surgically assisted palatal expansion with attachment of Hyrax expansion appliances was used as treatment protocol for correction of MTD and the increase of both the nasal volume and the intern nasal space. In the current study, all patients were selected from a group of adult and otherwise healthy subjects to minimize both the adverse effects of aging and the possibility of systemic disease that might interfere with the healing process.

In the current study it is unnecessary to carry out osteotomy in the pterygoid processes. To minimize the surgical trauma, less invasive procedures combined with osteotomies in the bilateral zygomatic maxillary crest and median palatine suture were performed by Kaban and Pogrel et al.(11-13). Endoscope is minimally invasive, diagnostic medical procedure. It is used to examine the inferior surfaces of an organ or tissue and allows visualization of body cavities. The nasal endoscope is a medical device consisting of a thin rigid tube with fiber optic cables for bringing in light.it passes through the nostril to examine the nasal passages, structures and sinuses. it is safe and low risk procedure. Endoscope allows for direct colorful visualization of the nasal cavities. This is especially important in the postsurgical patient when the nasal cavities anatomy is altered ${ }^{(14)}$. There were no significant differences in the changes in the left vs the right nasal cavities in any measurements (areas of ANC and PNC) at the various time intervals. This finding was supported in a study by Bell that discussed the variability in the pattern of expansion depending on Percentage changes in volume from T1 to T2 to T3. ${ }^{(15)}$ We also found that the PNC increased more than the ANC, even though the intermaxillary suture split with the SARME appliance decreases posteriorly. A possible explanation is that the PNC are larger in area than the ANC. This is in agreement with the results of Jacqueline Palaisa a et al. ${ }^{(16)}$ 


\section{CONCLUSION}

According to the results of the study, it could be stated that nasofibroscopy has a good role in evaluation of nasal airways with surgically assisted rapid maxillary expansion in adult

\section{REFERENCES}

1. Thomas M. and Doris G E. Maxillary transverse deficiency. American Journal of Orthodontics and Dentofacial Orthopedics, 2000; 1:540-4.

2. Zemann W, Schanbacher M, Feichtinger M, Linecker A, Kärcher H. Dentoalveolar changes after surgically assisted maxillary expansion: a three-dimensional evaluation, Oral Surg Oral Med Oral Pathol Oral Radiol Endod, 2009; 107: 36-42.

3. Ana Célia F, Samuel P. Enlargement of the Pharynx Resulting from Surgically Assisted Rapid Maxillary Expansion. Int J Oral Maxillofac Surg 2016; 74:369-79.

4. Landes CA, Laudemann K, Petruchin O, Ludwig B. Comparison of bipartite versus tripartite osteotomy for maxillary transversal expansion using 3-dimensional preoperative and post expansion computed tomography data. J Oral Maxillofac Surg 2009; 67: 2287-301.

5. Lobato HI, Machado SM, Ribeiro SM. Airway flow and audiologic ability evaluation after rapid maxillary expansion case report. Int J Pediatric Otorhinolaryngology Extra 2010; 5:89-90.

6. Chiari S, Romsdorfer P, Swoboda H, Ban-tleon H. Effects of rapid maxillary expansion on the airways and ears apilot study. Eur J Orthod 2009; 31: 135-41.
7. Edmilsson P J, Luiz G. Evaluation of the effect of rapid maxillary expansion on the upper airway using nasofibroscopy: Case report and description of the technique. Dental Press J Orthod 2011; 16:81-9.

8. Lanigan DT, Mintz SM. Complications of surgically assisted rapid palatal expansion: review of the literature and report of a case. J Oral Maxillofac Surg 2002; 60:104-10.

9. Bays RA, Greco JM. Surgically assisted rapid palatal expansion: An outpatient technique with long-term stability. J Oral Maxillofac Surg 1992; 50:110-13.

10. Verstraaten, J. A systematic review of the effects of bone borne surgical assisted rapid maxillary expansion. J Cranio Maxillofac Surg 2010; 38, 166-74.

11. Pogrel MA, Kaban LB, Vargervik K, Baumrind S. Surgically assisted rapid maxillary expansion in adults. Int $\mathbf{J}$ Adult Orthodon Orthognath Surg 1992; 7:37-41.

12. Freitas RR, Gonçalves AJ, Moniz NJ, Maciel FA. Surgically assisted maxillary expansion in adults: prospective study. Int J Oral Maxillofac Surg 2008; 37:797-804.

13. Kaban LB. Surgical orthodontic correction of transverse maxillary deficiency: a simplified approach. Plast Reconst Surg 1984; 73:67-68.

14. Aukema AA, Fokkens, WJ. Chronic rhinosinusitis: management for optimal outcomes. Treat Resp Med 2004; 3:97-105.

15. Bell RA. A review of maxillary expansion in relation to the rate of expansion and patient's age. Am J Orthod 1982; 81:32-7.

16. Jacqueline P, Peter N, Chris M. Use of conventional tomography to evaluate changes in the nasal cavity with rapid palatal expansion. Am J Orthod Dentofacial Orthop 2007; 132:458-66. 